\title{
CONOCIMIENTO DIDÁCTICO DEL CONTENIDO SOBRE FOTOSÍNTESIS DE DOS PROFESORES DE LOS GRADOS SEXTO Y NOVENO DE EDUCACIÓN BÁSICA SECUNDARIA DE UN COLEGIO PRIVADO EN BOGOTÁ-COLOMBIA
}

\section{PHOTOSYNTHESIS PEDAGOGICAL CONTENT KNOWLEDGE OF TWO TEACHERS FROM SIXTH AND NINTH GRADE OF BASIC SECONDARY FROM A PRIVATE SCHOOL IN BOGOTÁ-COLOMBIA}

\begin{tabular}{|l|}
\hline Recibido: 13-04-2012 \\
\hline Aceptado:18-07-2012 \\
\hline
\end{tabular}

\section{Por: Sonia Esmeralda Garnica ${ }^{1}$ Robinson Roa Acosta ${ }^{2}$}

\section{Resumen}

Este artículo presenta los resultados obtenidos en un estudio que tuvo como objetivo analizar el conocimiento didáctico del contenido sobre la fotosíntesis (CDCF) de dos profesoras de Biología, nivel de educación de secundaria en un colegio privado de Bogotá (Colombia). El enfoque fue interpretativo de tipo cualitativo. El método consistió en un estudio de caso y se utilizó la entrevista semi-estructurada, el cuestionario y el registro fílmico como técnicas de recolección de información. Se configuraron los elementos del CDCF de acuerdo al análisis de investigaciones relativas a la enseñanza de la fotosíntesis y al conocimiento didáctico del contenido. Se encontró que hay diferencias en el CDCF de las profesoras de cada uno de los grados analizados. Además, que unos elementos del CDCF permiten construir y desarrollar otros y es necesario conocerlos para dar cuenta del conocimiento que permite a las profesoras enseñar la fotosíntesis.

\section{Abstract}

This paper presents obtained outcomes in a study that had the goal of analyze the Photosynthesis Pedagogical Content Knowledge of two Secondary Biology teachers, in a private school in Bogotá (Colombia). The approach was interpretative and qualitative type, the method consisted in a study case and it used the semi-structured interview, the questionnaire and the filmic record as a collecting information techniques It found that there

\footnotetext{
${ }^{1}$ Magister en Educación. Universidad Pedagógica Nacional. Lic. en Química y Biología Universidad de la Salle.

${ }^{2}$ Magister en Educación Universidad Pedagógica Nacional. Lic. en Biología Universidad Pedagógica Nacional
} 
are differences in the teachers' Photosynthesis Pedagogical Content Knowledge of each tested grade. Also that the elements of the PPCK let to build and develop others and they are necessary to know them for the knowledge that empowers teachers in the photosynthesis teaching process.

Palabras clave: conocimiento didáctico del contenido, fotosíntesis, enseñanza, conocimiento profesional del profesor de ciencias.

Keywords: Photosynthesis, Photosynthesis Pedagogical Content Knowledge, Science Teacher Profesional Knowledge.

\section{Introducción}

En las aulas de clases de los profesores de ciencias, circulan variados conocimientos que tienen diferentes niveles de complejidad y en la realidad muchas veces son ignorados o no son tenidos en cuenta para la enseñanza. Un tema de gran importancia para los profesores de ciencias naturales (profesores de Biología, Física y Química) es el de la fotosíntesis.

Este concepto tiene un lugar importante en la enseñanza de las ciencias naturales, en tanto puede ser entendido como estructurante de otros conceptos tales como el de ecosistema y ambiente, sin negar que puedan encontrarse otros conceptos biológicos asociados a estos (célula, planta, respiración, nutrición, entre otros); los referentes teóricos para abordar su enseñanza tiene relación, aparte de la Biología, con la Química, al igual que tiene estrecha relación con lo económico y político en términos de la producción de energía para los países (biocombustible, producción de alimentos). De hecho, se puede decir que del proceso de fotosíntesis depende parte de la vida y lo vivo en el planeta tierra. En este sentido, la fotosíntesis puede ser tratada en la enseñanza de forma interdisciplinar (González, 2003; Stone, 1998; Cañal, 2004).

Teniendo en cuenta lo anterior, además de que el conocimiento del profesor tiene incidencia en lo que hace en el aula de clase y en los resultados de aprendizaje de los estudiantes, surge el interés por analizar el plan de estudios y los grados (sexto y noveno) donde se aborda el concepto fotosíntesis en el colegio privado San Marcos, para analizar el conocimiento sobre la fotosíntesis de dos profesores de Biología. Para esto se tomaron como fundamento teórico los elementos que configuran el conocimiento didáctico del contenido (CDC), entre los que se encuentran: conocimiento de los estudiantes, las formas de evaluar, las estrategias didácticas y metodológicas para enseñar el contenido, los propósitos de la enseñanza, las dificultades y limitaciones en la enseñanza, el currículo, experiencia y antecedentes académicos del profesor. Todos estos elementos confluyen y se interrelacionan en la enseñanza de un contenido específico, a la vez que permiten conocer la manera como enseña el profesor (Loughran, 2001; Charrier, 2005, 2009; Valbuena, 2007). 
El desarrollo del trabajo consistió en la selección y establecimiento de los elementos pertinentes para caracterizar y analizar el conocimiento didáctico del contenido sobre fotosíntesis (CDCF) de los profesores, y con base en esto diseñar instrumentos (entrevista y cuestionario) para recolección de datos. Se hizo registro videográfico de tres clases, se tuvo en cuenta las planeaciones de clase y guías de trabajo de las profesoras que permitieron obtener la información requerida para el análisis de sus CDCF. Los datos recogidos de cada una de las profesoras se analizaron individualmente y posteriormente se compararon, a partir de esto se establece que las profesoras tienen pocos conocimientos sobre la historia y el origen del concepto, que los proyectos de aula y las prácticas de laboratorio son las estrategias preferidas por las profesoras y que el uso de modelos o simulaciones puede llegar a ser una dificultad, al igual que la falta de interdisciplinariedad; los elementos propuestos para el CDCF en esta investigación aportaron para el análisis de los datos, no obstante, de lo complejo que resulta realizar análisis en su conjunto.

\section{Referentes generales sobre el conocimiento profesional del profesor}

Las investigaciones relacionadas con el conocimiento profesional del profesor se han profundizado a partir de la publicación de Lee Shulman en 1986: Those who understand: Knowledge growth in teaching, en la cual usó el concepto Pedagogical Content Knowledge (PCK) para referirse a ese conocimiento que el profesor tiene y que facilita o dificulta el la enseñanza del contenido de un tema específico; conocer las concepciones de los alumnos y las formas para hacer posible que otros comprendan los contenidos de enseñanza.

Se puede decir entonces que fue a partir de este autor que se han desarrollado de manera prolífica investigaciones con profesores de diferentes áreas del saber (Carlsen, 1987; Grossman, 1990; Loughran, 2001; Valbuena, 2007). Esto ha permitido ampliar, analizar, proponer y reorganizar los componentes del conocimiento del profesor.

Para Bromme (1988: 20) el conocimiento profesional del profesor es el que utiliza en su práctica cotidiana. Este requiere la interrelación de diferentes tipos de conocimientos, como son: los específicos de la asignatura que se enseña (conocimientos disciplinares), los de la didáctica específica, el pedagógico y el metaconocimiento.

Para autores como Porlán (1997) el conocimiento profesional es el que el profesor ha elaborado a partir de su formación académica y su experiencia. Por su parte, Tardif (2004) señala que el saber profesional está, en cierto modo, en la confluencia entre varias fuentes de saberes provenientes de la historia de vida individual, de la sociedad, de la institución escolar, de los otros agentes educativos, de los lugares de formación. Cuando se movilizan estos saberes en las interacciones diarias en el aula, convergen hacia la intención educativa del momento. 


\section{Conocimiento didáctico del contenido}

Marcelo (1999) fue el primero en aclarar que lo que se denomina conocimiento didáctico del contenido (CDC), es equivalente al utilizado por los investigadores norteamericanos y anglosajones: Pedagogical Content Knowledge (PCK). Es importante dicha aclaración, ya que para el caso hispanoparlante, existen autores que se refieren al CDC y al PCK, mientras que en el ámbito anglo solo se refieren al PCK

En la revisión bibliográfica realizada sobre el CDC se puede encontrar que algunos autores (Shulman, 1986; Grossman, 1990; Gess-Newsome, 1999; Carlsen, 1999; Magnusson, Krajcik y Borko, 1999) han presentado componentes comunes del conocimiento profesional, entre ellos: conocimiento del contenido, conocimiento pedagógico, conocimiento didáctico del contenido y conocimiento del contexto.

Para ellos el CDC se refiere al conocimiento que se necesita para poder transformar los contenidos disciplinares, con el fin de hacerlos más comprensibles a los alumnos y facilitar así su aprendizaje. Se trata entonces de hacer que los contenidos disciplinares sean enseñables y aprendibles, para facilitar la apropiación por parte de los estudiantes de una disciplina determinada.

Además, coinciden en que es el componente que mayor incidencia tiene en las acciones de enseñanza en el aula de clase y enfatizan en conocer las concepciones y los intereses de los alumnos para la definición y estructuración de los contenidos curriculares y de las estrategias de enseñanza.

Shulman $(1986,1987)$ le asigna al PCK la categoría más distintiva del conocimiento que identifica al profesor, destacando que es en este conocimiento donde se integran y transforman los diferentes componentes involucrados en la enseñanza. Caracterizó el PCK por la particularidad de: comprender los aspectos que facilitan o dificultan el aprendizaje del contenido de un tópico específico; conocer las concepciones de los alumnos de diferentes edades y procedencia acerca de un contenido en particular; utilizar estrategias, tales como "analogías, ejemplos, explicaciones y demostraciones, es decir formas de representación y formulación para hacer posible que otros comprendan los contenidos de la enseñanza.

Valbuena (2007) indagó y analizó las concepciones disciplinares y didácticas de profesores en formación en la Universidad Pedagógica Nacional de Colombia sobre los diferentes constituyentes del conocimiento profesional del profesor de Biología, y concretamente del conocimiento didáctico del contenido biológico.

Charrier (2005, 2009), desarrolló dos investigaciones alrededor del CDCF. En la primera, estudió las concepciones de los profesores sobre la enseñanza y 
aprendizaje de la fotosíntesis utilizando cinco categorías, en la segunda, hizo una caracterización del CDCF que presentaban dos profesores de secundaria.

En relación con el CDC algunos autores refieren a la dificultad para identificarlo, ya que se trata de un conocimiento tácito construido internamente y difícil de evaluar. Su identificación es un proceso complejo y difícil, ya que no está asociado con una determinada lección y se puede llegar a reconocer luego de un período relativamente largo de tiempo (Lorenzo y Farré, 2009).

Para establecer algunos de los elementos de análisis del CDCF para esta investigación, se tomaron como referencia los presentados por Loughran (2001), Valbuena (2007) y Charrier (2005, 2009). Los autores coinciden en elementos comunes del CDC tales como: ideas de los alumnos, contenido por enseñar, evaluación de los aprendizajes, estrategias metodológicas de enseñanza. Algunos elementos como finalidades de la enseñanza y dificultades de la enseñanza no son comunes en todos. Es notable que algunos elementos son específicos de las investigaciones, como por ejemplo los estudiantes (indisciplinados, falta de interés, trabajo grupal), currículo y fuentes del currículo (Charrier, 2009). Finalmente es de resaltar que solo un autor (Valbuena, 2007) incluye las dificultades de la enseñanzaaprendizaje.

Esta investigación recoge algunos de los elementos presentados por los autores antes mencionados, en lo que sigue se abordan cada uno de los elementos de acuerdo a como son entendidos en este estudio, estos corresponden a los ocho descritos brevemente en la Tabla 1.

1) Conocimiento acerca de los estudiantes. Este elemento se refiere a las ideas previas, su uso, aprendizaje y comportamiento de los estudiantes. Según Osborne (1995: 178) "los estudiantes siempre tienen ideas previas que influyen en el pensamiento sobre cualquier tema". El comportamiento define el curso del desarrollo de una clase y puede determinar el aprendizaje de un conocimiento específico. 2) Conocimiento acerca del contenido disciplinar. Contempla los "contenidos disciplinares que sabe el profesor y que se enseñan, así como la forma como están organizados en estructuras sustantiva y sintáctica", según la clasificación de Joseph Schwab (1978, citado en Shulman, 1986: 9). Además sobre el contenido a enseñar incluye el porqué es importante, los conceptos con que se relaciona, las dificultades del concepto, la historia y origen del concepto. 3) Formas de evaluar el aprendizaje del concepto. Se entiende como la manera en la cual el profesor evalúa el aprendizaje de los estudiantes y las intenciones que persigue con la evaluación. 4) Estrategias didácticas para enseñar el concepto. Consisten en la manera como se han de presentar los contenidos a los estudiantes en el momento de la enseñanza, tales como metáforas, analogías, modelos, experimentos y explicaciones (Grossman, 1990). La labor del profesor implica reconstruir específicamente el saber que se enseña, media una modificación epistemológica del marco conceptual original. La trasposición didáctica indica que el paso del saber sabio al saber enseñado nunca es directo. En ocasiones la trasposición es tal, que a veces nos 
lleva a la creación de objetos nuevos, que no tienen, como tales, equivalentes en el saber erudito (Astolfi, 2001). 5) Propósitos de la enseñanza del concepto. Se refiere a los objetivos que el profesor propone para la enseñanza de un concepto. Los profesores están en desacuerdo frente al hecho de preparar a los estudiantes para que aprueben evaluaciones, pero se debería propender por dar los elementos educativos, pedagógicos y didácticos, para contribuir en la formación general del individuo, la aplicabilidad de la Biología escolar en la vida cotidiana de los estudiantes, la conservación de la naturaleza, el apropiado uso de los recursos naturales y el aprendizaje de lo vivo entendiéndolo como un sistema natural (Valbuena, 2007). 6) Dificultades y limitaciones en la enseñanza del concepto. Hace referencia a los factores que intervienen y podrían generan obstáculos en la enseñanza de un concepto. Algunos estudios han encontrado que la dificultad principal en la enseñanza del concepto es que los estudiantes no dominan la distinción entre los conceptos: elementos, compuestos y conservación de la materia, lo que les impide comprender la presencia del carbono en el gas carbónico o de nitrógeno en las sales minerales e intercambios con la atmósfera. Por otro lado, está la presencia de aprendizajes anteriores que interfieren, por ser incorrectos, en el aprendizaje de las ideas o destrezas (Wandersee, 1983). 7) Currículo. Se refiere a la organización, estructuración y enfoque de enseñanza que orientan el desarrollo de las clases. A continuación se expone lo que para Díaz (2010) se relaciona con el currículo. Hay unas dimensiones del currículo que se relacionan con la enseñanza, como son los contenidos escolares, la metodología y la evaluación. 8) Conocimiento experiencial del profesor. Hace referencia al repertorio de experiencias profesionales y pedagógicas que poseen los profesores que pueden influir en sus referentes de enseñanza (Loughran, 2001). Desde su saber el profesor debe apoyar al estudiante a construir el conocimiento y a ubicarse como actor crítico de su entorno.

\section{Aproximación al proceso de fotosíntesis}

Para Canguilhen (1988) los conceptos son producto de la confrontación de nociones históricamente definidas, se ha ido estructurando debido a acontecimientos significantes que sitúan el concepto biológico según historia.

En cuanto a la historia de la fotosíntesis, se puede decir que comenzó hace 300 años con las investigaciones de Van Helmont y aún el problema no está resuelto. A principios del siglo XVII, cuando comenzó el estudio experimental de la fisiología vegetal había muy poca evidencia para mostrar cómo ocurrían las reacciones químicas. Los hombres notaban que algunas sustancias entraban a las reacciones químicas y otras sustancias se producían en ellas.

En lo que sigue, se muestran los hechos que presenta Baker (1970) en su libro Biología e investigación científica y se complementa con otras referencias que confirman dichos acontecimientos. 
En 1702, Georg Ernest Stahl (1660-1734), desarrolló la teoría del flogisto para poder explicar la combustión. Jan Baptista Van Helmont (1577-1644), uno de los personajes más sobresalientes en los inicios del desarrollo del concepto fotosíntesis, introdujo entre los hombres dedicados a la Botánica la creencia de que el agua había sido transmutada en madera, tesis aceptada por la sociedad del momento; el agua por sí sola era el elemento que mantenía la vida de la planta. En la última parte del siglo XVII el desarrollo del microscopio llevó al descubrimiento de que las hojas de las plantas tienen muchas aberturas (estomas) en su superficie, a partir de lo cual surgió la pregunta si era posible que estos permitieran el intercambio de sustancias entre la planta y la atmosfera. De aquí surgió una nueva pregunta: tal vez el agua no es la única relacionada con la nutrición de las plantas. Fue Stephen Hales quien concluyó que las plantas toman algo del aire y posteriormente Lavoisier echó abajo la teoría del flogisto e incluyó el suelo como fuente de pequeñas cantidades de tierra. Luego Priestley realizó una serie de experimentos relativos a la combustión y a la respiración, concluyendo que las plantas podían invertir el proceso respiratorio de los animales y tenían un efecto purificador sobre el aire. En 1817, dos químicos franceses, Pelletier y Caventou, aislaron la sustancia verde de las hojas y la llamaron clorofila. Otro acontecimiento en la historia de la fotosíntesis tuvo lugar en 1845 cuando Robert Mayer, enunció que las plantas transforman la energía solar en energía química.

En 1885 George Heves abrió nuevos caminos experimentales usando isótopos radioactivos para trazar vías por las que se movían los materiales de un lado a otro en las plantas. Así se comprobó que el origen del oxígeno era el agua. En 1905 Blackman sugirió que la fotosíntesis procedía según un mecanismo de dos pasos y que implica una reacción fotoquímica o a la luz (fase luminosa), y una reacción no fotoquímica o en la oscuridad (fase oscura) En 1948, continuando estos estudios con isótopos radiactivos, Melvin Calvin (Premio Nobel de 1961) introdujo el isótopo carbono 14 y realizó estudios basados en las reacciones de oscuridad. Concluyeron que el carbón radiactivo iba a formar parte de las moléculas de glucosa.

Actualmente se sabe que los organismos con clorofila son capaces de captar la energía del sol para transformar la materia mineral en materia orgánica. El proceso de captación y transformación de la energía solar en química se conoce con el nombre de fotosíntesis.

La fotosíntesis es llevada a cabo por plantas, algas verdes, líquenes y bacterias foto sintetizadoras (Audesirk, 2001). Los elementos necesarios para el proceso son: energía, dióxido de carbono $\left(\mathrm{CO}_{2}\right)$ y agua. La energía proviene del sol y es absorbida por un pigmento verde llamado clorofila. El dióxido de carbono está en el aire y se introduce en las plantas a través de las estomas, diminutos poros del envés de las hojas. El agua es absorbida del suelo por las raíces (Asimov, 1980). 


\section{Enseñanza de la fotosíntesis}

Teniendo en cuenta que los procesos de fotosíntesis y respiración celular han hecho parte de los lineamientos curriculares establecidos por el Ministerio de Educación Nacional de Colombia para el área de Ciencias Naturales (MEN, 2006), desde preescolar es evidente que existen problemas alrededor de la enseñanza del proceso de fotosíntesis. Diversos investigadores han tratado de determinar el grado de dificultad que presenta para los profesores. Así por ejemplo, Johnstone y Mahmoud (1980) encontraron varios conceptos, entre ellos la conversión energética en la fotosíntesis y la respiración. Test y Wewards (1980), se refieren a la fotosíntesis como concepto científico de gran dificultad didáctica. Situación que es confirmada por Finley (1982), quien hizo un estudio con profesores en ejercicio e identificó qué contenidos en asignaturas especificas son importantes para enseñar y difíciles de aprender, siendo la fotosíntesis un concepto seleccionado como de los más importantes y uno de los más "difíciles".

Estudios realizados con profesores, muestran hasta qué punto es generalizada la presencia de errores conceptuales en cuanto a la fotosíntesis de las plantas verdes. Se encontró que factores como la luz, dióxido de carbono, clorofila, agua y sales minerales no eran considerados imprescindibles por los profesores para que se realice el proceso de la fotosíntesis de las plantas verdes (Gené, 1984), por tal motivo se planteó la necesidad de realizar estudios más profundos al respecto.

\section{Materiales y métodos}

Es de aclarar que la estructura del servicio de educación formal colombiano es aquel que se imparte en establecimientos educativos aprobados, en una secuencia regular de ciclos lectivos, con sujeción a pautas curriculares progresivas, conducentes a grados y títulos. Los niveles de formación de la educación formal son: educación preescolar, que comprende mínimo un grado obligatorio, educación básica, con una duración de nueve grados (básica primaria: cinco grados y básica secundaria: cuatro grados, y educación media, con una duración de 2 grados (10․ y 11․) Culmina con el título de bachiller (MEN, 2008). Esta investigación se llevó a cabo en básica secundaria (grados sexto y noveno).

Esta investigación se desarrolló en el enfoque interpretativo, el cual según Arnal (1992), se orienta a descubrir y comprender la realidad social y el contexto educativo.

En un primer momento se realizó una revisión bibliográfica relacionada con el problema de investigación, se seleccionó y sistematizó.

En un segundo momento se hizo el diseño de la investigación, que básicamente consistió en escribir los antecedentes, predeterminar los objetivos a la luz del problema y trazar la configuración de los referentes conceptuales en donde se sustentó y profundizó los diferentes conceptos y temas que son punto de referencia 
En un tercer momento se establecieron las preguntas tanto para la entrevista como para el cuestionario, a partir del conocimiento de los autores y de otros trabajos relacionados (Gómez, 2000). Posteriormente se hizo la correspondiente validación con expertos de la línea de investigación Conocimiento Profesional del Profesor de Ciencias de la Universidad Pedagógica Nacional $^{3}$ y luego se aplicaron los instrumentos antes de comenzar el tercer periodo académico, en donde se desarrolla el tema de fotosíntesis. Finalmente se aplicó la entrevista con la cual se indagaron realidades múltiples. $Y$ con el cuestionario se pretendieron respuestas de mayor precisión. Se hicieron tres filmaciones de clases para cada una de las profesoras. Como insumos extra se contó con la planeación previa de las clases.

En un cuarto momento, luego de la recopilación de la información, se empezó la codificación de la información que fue específica para cada uno de los instrumentos y elementos del CDCF. Para el procesamiento de datos se hizo la transcripción total y fidedigna de las respuestas correspondientes a las preguntas planteadas en la entrevista y el cuestionario, las grabaciones de las clases, la planeación y las guías de clase. La organización de los datos se hizo acorde al elemento y posteriormente se redactaron los descriptores, estos consistieron en la síntesis de los datos recogidos para cada una de las profesoras.

Para los análisis de resultados se utilizaron en ocasiones los fragmentos de explicaciones de la profesora y/o discusiones entre la profesora y los estudiantes tomados directamente de las transcripciones, en otros casos se usaron los descriptores, siendo este último un reflejo explícito de los que las profesoras dijeron, hicieron o escribieron. En el análisis aparecen varios códigos al final de la información, lo que permite identificar si es un fragmento directo de las transcripciones, un descriptor, su procedencia, la profesora, el instrumento, el elemento del CDCF, etc. La Tabla 2 muestra los insumos sistematizados, los códigos y los elementos indagados para cada instrumento durante la investigación.

Tabla 2. Codificación y sistematización de la información recogida para los elementos del CDCF

\begin{tabular}{|c|c|c|c|}
\hline INSTRUMENTO & $\begin{array}{c}\text { CÓDI } \\
\text { GO }\end{array}$ & INSUMOS & ELEMENTO INDAGADO \\
\hline Entrevista & $\mathrm{I}$ & 1 & $\mathrm{E}-1 \mathrm{y} \mathrm{E}-8$ \\
\hline Cuestionario & $\mathrm{C}$ & 1 & $\mathrm{E}-2,-3,-4,-5,-6,-7$ \\
\hline Planeación & $\mathrm{P}$ & 3 & $\mathrm{E}-3,-4,-5$ \\
\hline Guías & $\mathrm{T}$ & 2 & $\mathrm{E}-3,-4$ \\
\hline $\begin{array}{c}\text { Grabación de } \\
\text { clases }\end{array}$ & $\mathrm{G}$ & 3 & $\mathrm{E}-1,-2,-3,-4,-5,-6,-7,-8$ \\
\hline \multicolumn{2}{|c|}{ Caso } & Código \\
\hline \multicolumn{2}{|c|}{ Profesora Ana } & A \\
\hline \multicolumn{2}{|c|}{ Profesora Beatriz } & Código \\
\hline \multicolumn{2}{|c|}{ Otros } & \\
\hline
\end{tabular}

\footnotetext{
${ }^{3}$ Es de aclarar que dicha validación se realizó, pero para la aplicación de las preguntas a las profesoras no se tuvo a tiempo debido a situaciones académicas propias del colegio donde se realizó la investigación.
} 


\begin{tabular}{|c|c|}
\hline Unidades de Análisis & U \\
\hline Descriptores & $\mathrm{D}$ \\
\hline Pregunta & $\mathrm{Q}$ \\
\hline Respuesta & $\mathrm{R}$ \\
\hline
\end{tabular}

Luego de organizar, codificar y sistematizar los datos se hizo un análisis de la información recogida para las profesoras, que se presenta a continuación.

\section{Resultados y análisis}

En este apartado, inicialmente se presentarán las características de las profesoras y posteriormente se mostrarán los análisis conjuntos para las dos profesoras. Es de aclarar que el enfoque metodológico que orienta el quehacer de las profesoras en el aula de clase es de tipo constructivista y utilizan como estrategia la enseñanza para la comprensión, siendo este el que direcciona a todo el área de Ciencias Naturales en el colegio privado San Marcos. Además es de aclarar que cuando se hace referencia a licenciado es para el caso de profesores que han sido formados desde el pregrado como profesores.

\section{Características generales de las profesoras}

En cuanto a la profesora de los grados sextos, Ana, tiene 27 años y de experiencia docente cuatro años, ha trabajado la mayor parte del tiempo en secundaria y actualmente orienta la asignatura de Ciencias Naturales (en dos periodos orienta Biología, en uno Física y en otro Química) en los grados quintos, sextos y séptimos, licenciada en Química.

En cuanto a su formación pedagógica asegura:

A.: "He hecho curso de actualización y en la misma práctica he aprendido algunos procesos que permiten enseñar mejor".

La profesora Beatriz tiene 28 años y tiene experiencia de cinco años, ha trabajado en colegios privados, la mayor parte del tiempo en secundaria. Actualmente orienta la asignatura de Biología en los grados octavos y novenos. Estudió durante cinco años y obtuvo el título de bióloga aplicada.

Entre las características que tiene como profesora, ella menciona que se deben a:

B.: “....me gusta enseñarle a los niños porque me gusta que aprendan lo que a mí me gusta".

Aunque no es licenciada, asegura que ha sido la experiencia lo que le ha permitido formarse.

Para el análisis que sigue, se tuvo como objetivo relacionar la referencia teórica de los elementos del CDCF con los resultados encontrados en cada uno de los 
instrumentos utilizados con los que se recogió información acerca del CDCF de las profesoras Ana y Beatriz. Es importante resaltar que se presentan de las dos profesoras los resultados más relevantes, ya que en la investigación se hicieron análisis individuales como conjuntos.

El conocimiento acerca de los estudiantes que tienen las profesoras da cuenta de sus propias vivencias y experiencias como estudiantes y como profesoras. Se evidenció por medio de la comparación entre lo que las profesoras dijeron y lo que los estudiantes expusieron en el aula de clase: que las ideas previas que proponen las profesoras se relacionan con temáticas que muchos estudiantes no conocen a profundidad. Por ejemplo:
A.:- La fotosíntesis se puede relacionar con la respiración en el humano, con toda la respiración y con toda la obtención de energía que los seres vivos necesitan. $A, I, E-1 R-1^{4}$

Además, en el siguiente apartado se hace evidente que es la profesora quien lleva a los estudiantes a relacionar la fotosíntesis con algunos de los temas que ella nombra en la entrevista (obtención de energía que los seres vivos necesitan), por ejemplo:
A.:- ¿Qué pasaría en nuestro planeta si no tenemos plantas?
E.:- Nosotros morimos porque las plantas dan oxígeno.
A.:- Dan oxígeno, exacto y ¿qué más? A, G-1, E-1 p3

Se puede ver que es la profesora quien plantea la situación y orienta la discusión respecto de la relación que tienen las plantas con la producción de oxígeno .

Respecto de los errores de los estudiantes, las profesoras Ana y Beatriz enuncian en la entrevista que:

A.:-Que solo el oxígeno se convierte en azúcar, que no asocian factores como el dióxido de carbono, no asocian reacciones del día y de la noche. A, I, E-1, R-2.

B.:- El error más común es que piensen que existe solamente una forma de realizar el proceso o que las plantas verdes son las únicas que realizan la fotosíntesis. B, I, E-1, R-2.

En ocasiones los errores mencionados no corresponden con el grado, con el nivel de pensamiento ni con las acciones de producción concretas con las que cuentan sus estudiantes, por lo que se debe planear a lo largo de los grados de escolaridad secuencias graduales en cuanto a complejidad y contenido, además contar con la interdisciplinaridad para que los estudiantes relacionen con el contenido de otras

\footnotetext{
${ }^{4}$ Codificación para identificar: profesora, tipo de insumo, elemento y página de procedencia del documento de transcripción.
} 
asignaturas que les permita construir modelos que reflejen los propuestos por la comunidad científica actual.

Respecto del conocimiento que tienen las dos profesoras sobre el aprendizaje de los estudiantes sobre la fotosíntesis, ambas refieren la enseñanza como forma de aprendizaje, ambas presentan dificultades para explicitar la forma de aprendizaje. Es importante conocer cómo los estudiantes interpretan, comprenden y van construyendo las relaciones entre lo que ven y lo que los profesores explican.

Por ejemplo, en relación a la pregunta ¿cómo aprenden los estudiantes?, la profesora dijo que los estudiantes lo hacen por medio de muchas actividades como:

- Revisión de conceptos.

- Revisar contenidos en libros y artículos.

- Experimentación.

- Trabajos de investigación. B,I,E-1,R-5

En relación con las ideas previas, Pozo (1987) propone que lo primero que se debería hacer, es estimar en cualquier aula, cuales pueden ser los puntos predominantes, posteriormente se puede ayudar a clarificar y si es necesario reformular sus ideas. Ana dijo lo siguiente al respecto:

A.:- Después de que indago las ideas previas de acuerdo a los resultados que obtuve, pues generalmente intento hacer una revisión o un repaso de los conceptos como los que dije de célula vegetal, pues para que ellos recuerden que es un cloroplasto y todo esta partes, hago un repaso basándome en los conceptos que revise en ellos. A, I, E-1, R-4.

B.:- Luego de indagar las ideas previas las clasifico y determino las relevantes o fundamentales, aclaro los conceptos, reviso y repaso para profundizar en lo que les llama la atención. B, I, E-1, R-4

Cabe indicar en este punto que el manejo que hacen de las ideas previas se relaciona con el elemento estrategias para la enseñanza del concepto, ya que las profesoras utilizaron diferentes técnicas para identificar las ideas previas de los estudiantes, el diseño de modelos, el procedimiento experimentales y simulaciones sobre las fases de la fotosíntesis, entre otras, exigieron que los estudiantes utilizaran lo que ya sabían, reforzaran o buscaran información para verificar dichos conocimientos.

Las profesoras usan las ideas previas para determinar si los estudiantes saben o no sobre el contenido, por ejemplo para procesos cognitivos como generación de expectativas apropiadas, se debe ser claro con los estudiantes dándoles a conocer las finalidades y el alcance del material y cómo manejarlos, de esta forma el estudiante sabe qué se espera de él al terminar de revisar el material. También ayudar a contextualizar sus aprendizajes y a darles sentido, activación de 
conocimientos previos como actividad focal, introductoria, discusiones guiadas, etcétera (Díaz, 2003).

Los contenidos disciplinares que sabe la profesora Ana se relacionan con la capacidad de las plantas de disminuir el $\mathrm{CO}_{2}$ y aumentar el $\mathrm{O}_{2}$ entendiéndolo como la base para la cadena alimenticia, mientras que la profesora Beatriz lo entiende como el proceso que transforma la energía lumínica en energía química.

A continuación se presentan las respuestas de las profesoras respecto de la pregunta ¿por qué es importante?
A.:-Porque a partir de la fotosíntesis se disminuye el $\mathbf{C O}_{2}$ y aumenta el $\mathbf{O}_{2}$ y además es la base para la cadena alimenticia, gracias a la producción de almidón, celulosa, glucosa y polisacáridos. A, C, E-2, R-7
B.:- Porque es el motor de muchos de los procesos de los seres vivos, es integrador de otros temas. , B, C, E-2, R-7

Dentro de los segmentos o diálogos obtenidos de las clases relacionados con la importancia de la fotosíntesis se encontraron los siguientes:
A.:- La fotosíntesis es para producir el oxígeno, pero las células de las plantas van a hacer la respiración también, que significa esto, ella misma va a tomar el oxígeno que ella misma produce y va a hacer el proceso de respiración. A, G-3, E-5, U-5
B.:- La fotosíntesis es el principal proceso para la planta porque usa la energía y la fuente más importante de energía es la luz solar, porque inclusive si no tiene luz solar, puede llevar a cabo la fotosíntesis con una lámpara pero no de igual manera como si tuviera toda la energía del sol. B, G-3, E-5, U-1.

Entre las razones que dan las profesoras sobre la importancia de la fotosíntesis, resaltan la producción de sustancias para los demás organismos y para favorecer algunas actividades económicas. Lo que podría relacionarse con sentido utilitarista y consumista de los recursos naturales. Los términos que usan las profesoras están en un marco teleológico, es decir asumen una causa final (Mayr, 2006). Es de destacar que las profesoras no refieren conocimientos históricos sobre la temática. Es importante tener en cuenta que muchos son los estudios que se han hecho en cuanto al proceso de construcción histórica del conocimiento sobre la fotosíntesis, lo que permite el acercamiento al proceso de creación científica y posibilita la contextualización del conocimiento, a comprender su evolución y las relaciones existentes con la ciencia, la tecnología, la sociedad y el ambiente (CTSA) (Quintanilla, 2010). 
Sobre el origen y la historia las profesoras no refieren ningún conocimiento. Es de resaltar que es necesario que el profesor conozca tanto el origen como los cambios históricos que han acontecido en torno al concepto a enseñar (Eisen et al, 1992; Carlsen, 1999; Gess-Newsome, 1999), y es necesario que el estudiante comprenda que es una construcción humana de tipo teórico y en la medida en que la ciencia avanza, se establecen cambios que posiblemente tienen impacto en la tecnología y la sociedad

No es clara una estructura específica del conocimiento disciplinar sobre fotosíntesis, ni un nivel de jerarquización del concepto fotosíntesis. Para ambos casos el contenido educativo se basa en temas e información expuestos por las profesoras, es posible que lo que direccione su que hacer en cuanto al contenido esté básicamente direccionado por los libros guía que utilizan en cada uno de los cursos.

Las profesoras refieren a las formas de evaluar para la enseñanza (actividades) pero no para el aprendizaje. Existe en el colegio donde las profesoras laboran un documento del área de Ciencias Naturales (Matriz de Evaluación) donde se establece el objeto y el nivel de referencia, delimitando los aspectos en particular que se deben evaluar, consta de unos criterios y descriptores de los desempeños que permitirían hacer un buen proceso de evaluación.

En primer lugar se les preguntó a las profesoras qué buscaban saber a través de la evaluación y Ana contestó:

A.:- Busco evaluar los conocimientos que los estudiantes tienen y como relacionan y aplican los conocimientos en situaciones cotidianas. A, C, E-3, $\mathrm{R}-13$

En cuanto a lo que Ana busca saber a través de la evaluación en las sesiones de clase, se registró lo siguiente en el documento de planeación de clase:

El profesor evaluará el trabajo y comportamiento. A, P-1, E-3, U-1.

Presenta una ambigüedad en lo que formuló desde el documento de planeación, ya que el trabajo realizado y el comportamiento son criterios muy amplios que presentados de esta manera no permiten concretar con certeza que es lo que en realidad busca evaluar.

Beatriz dijo lo siguiente:

B.:- A través de la evaluación busco saber como pueden los estudiantes enfrentarse a pruebas escritas con ciertos tipos de preguntas cuando la herramienta utilizada es un prueba escrita (evaluación bimestral), busco conceptos errados que se puedan aclarar con estudios de casos B,-1-2-3, E3, D-1 
En cuanto al nivel de desempeño, la profesora refiere a la enseñanza para la comprensión, que es la estrategia que se utiliza en el colegio y que establece unos parámetros para identificar en qué nivel se encuentra el estudiante de acuerdo a los resultados que obtiene en las diferentes formas de evaluación.

Aunque la evaluación diagnóstica es fundamental, no se da en la práctica, las profesoras utilizan diferentes estrategias como actividades, trabajos prácticos, participación en clase, interrogación oral, discusión en clase, toma de apuntes en el cuaderno, resúmenes para hacer una evaluación formativa y sumativa. La evaluación planteada en los documentos de planeación de clases en ocasiones difiere con lo que se hace en clase. En la práctica la profesora Ana evalúa el reporte de laboratorio, el taller y el cuaderno, la profesora Beatriz el trabajo en el cuaderno, un resumen, exposiciones orales, trabajo práctico. De todas las actividades desarrolladas por las profesoras se nota mayor eficacia en la ejecutada por la profesora Beatriz, quien propuso un trabajo práctico en casa: consiste en que los estudiantes hagan un planteamiento, desarrollo y análisis de una práctica relacionada con la fotosíntesis y los factores que intervienen en el proceso.

Es evidente que la evaluación se llevó a cabo en todo momento, algunas preguntas que se hicieron son parte de una evaluación formativa. La evaluación sumativa corresponde a otro tipo de actividades. Como lo plantea Álvarez (2007), en la evaluación en un proceso complejo y continuo que acompaña el desarrollo continuo de la actividad de enseñanza, esta concepción puede propiciar el desarrollo de evaluaciones más justas y orientadas por principios éticos que formen a los estudiantes, para constituirse como un proceso para aprender.

En cuanto a las estrategias didácticas, las profesoras refieren a las actividades que utilizan, no al procedimiento o secuencia.

Frente a la siguiente instrucción en el cuestionario: describa las maneras con las que enseñaría el concepto de fotosíntesis a sus estudiantes. Y ¿cuáles se pueden llevar a cabo en esta institución?, Ana contesto:

A.:- A través de una explicación teórica con modelos de la célula vegetal y el cloroplasto, a través de una presentación y un video de animación que ilustre la fotosíntesis, con un laboratorio de aplicación de conceptos de fotosíntesis, y todos ellos son aplicables en el colegio. A, C, E-4, R-16

Beatriz contestó que enseñaría el concepto de fotosíntesis a través de:

B.:-Estudios de caso, explicación, simulación o modelación, experimentación, toma de notas, resolución de preguntas. B, C, E-4, R-16

Presentan algunas diferencias, por ejemplo en el grado sexto se plantea una práctica de laboratorio y se refuerzan los contenidos en clase, mientras que en noveno la profesora valora mayoritariamente el trabajo de los estudiantes con quienes se 
alterna actividades de diseño, experimentación con sustentación oral y con la explicación de la profesora, prevalece en ambos casos un tipo de metodología expositiva con actividades como las prácticas de laboratorio y uso de modelos o simulaciones. Una secuenciación planteada con gran éxito ha sido el uso de un hilo conductor de la historia de la ciencia, ya que se ha demostrado que los profesores tienen un conocimiento que coincide con las teorías científicas que han surgido en el proceso histórico de desarrollo del concepto. (Eisen y Stavy, 1992; Quintanilla, 2010).

En las grabaciones de las clases se encontró que Ana utilizó el tablero para hacer dibujos, escribir definiciones, hacer mapas sencillos, también utilizó modelos a escala en icopor de la célula vegetal y guías de trabajo en clase:
A.:- Bien, miren aquí. Este es un modelo de la célula vegetal, como podemos ver, la forma de esta es cuadrada pero no todas son cuadradas [tiene el modelo en las manos] A, G-1- E-4, p2.

Muchos profesores consideran las ilustraciones, las gráficas, los resúmenes, como estrategias que permiten mejorar la codificación de la información nueva. Encuentran los modelos como una manera para potenciar y explicitar los conceptos científicos, hacer evidentes los procesos naturales e ilustrar lo que por diversos motivos es difícil ver directamente. Se han constituido como una herramienta muy útil en el campo de la enseñanza, ya que han permitido realizar una cantidad innumerable de representaciones, de la misma manera han sido analizados desde diferentes áreas del conocimiento para comprobar su validez en el campo científico (Chávez, 2010).

Respecto de los propósitos de enseñanza, aunque las dos profesoras tienen intenciones relacionadas con la comprensión de un contenido, existen variaciones.

Los objetivos que propone la profesora Beatriz para la enseñanza de la fotosíntesis parten de las intenciones que tiene cuando enseña. Al respecto ella dijo que se orientaba a:

- Motivar a los estudiantes.

- Generar curiosidad.

- Desarrollar tres competencias: identificar, indagar y explicar.

Las intenciones de la profesora Ana para enseñar la fotosíntesis, se orientan a dos cuestiones: la primera se relaciona con la importancia de las plantas para la vida y la segunda a comprender el procesamiento de $\mathrm{CO}_{2}$, de oxígeno y de glucosa que utiliza el humano. Para complementar esta idea, se dan algunos segmentos de las clases que vale la pena tener en cuenta:

A.: - Gracias a esto todos los seres vivos pueden existir. Bueno, ¿por qué? ¿Qué pasa por ejemplo? Piensen acerca de lo que pasaría si en nuestro planeta no tenemos plantas, ¿qué pasaría en nuestro planeta sino tenemos plantas? 
E.:- Nosotros morimos porque las plantas dan oxígeno. A, G-1, E-5, p3.

En otro momento de la clase Ana dijo:

A.:-¿Por qué es importante esto en una cadena? ¿Por qué es importante el cloroplasto? y ¿cuál es la importancia de estos en la vida de todos los seres vivos en el planeta? [Empieza a dibujar en el tablero]. A, G-1, E-5, p4.

La profesora Beatriz lo utiliza como una excusa para el desarrollo de competencias que aunque no reflejan coherencia desde lo que se planea y realmente se hace, en la práctica demuestra que las estrategias que ella aplica pueden permitir el cumplimiento de los objetivos que se plantean desde el área de Ciencias Naturales y Ambientalesde su colegio. Ambas le dan una finalidad y propósito al proceso de la fotosíntesis. A pesar de que las plantas producen su propio alimento, se le da un enfoque egocentrista (Brown, 2009), al referirse a las plantas como organismos que proveen a los humanos de oxígeno, pone a los humanos como beneficiarios de los procesos de las plantas y obvia el hecho de que las plantas son organismos con necesidades metabólicas que también dependen de otros animales.

En el elemento de dificultades y limitaciones en la enseñanza de la fotosíntesis se encontró que algunas se relacionan con la enseñanza-aprendizaje y otras con el currículo de la institución.

Para la profesora Ana una limitación muy grande es el material didáctico, ya que en la institución no hay uno acorde con estos cursos; ella dijo:

A.:- La limitación que encuentro en relación con el material didáctico es que es muy avanzado para el grado sexto o es muy básico, lo que dificulta el proceso de enseñanza. A, C, E-6, R-23

La profesora Beatriz encontró varios problemas. Entre ellos están:

- Las bases de física y química que tienen algunos estudiantes, no todos recuerdan los mismos conceptos.

- Es un contenido teórico que debe ser modelado (no es tangible).

- El tiempo.

Luego de confrontar todas las evidencias se encontró que las dificultades de enseñanza están relacionadas con el uso de modelos o simulaciones: los estudiantes deben tener claros conceptos biológicos para poder enfrentarse a un modelo. De esta forma el discurso del profesor se convierte en una herramienta: si este es fluido y claro seguramente proveerá de contextos para elaborar conexiones. Se pueden utilizar ejemplos para aclarar los mismos conceptos, tratando de concretarlos con objetos o situaciones que los ilustren. Los estudiantes poco a poco se deben apropiar 
del vocabulario para poder usar correctamente los modelos en el aprendizaje. El profesor puede contar con expresiones aclaratorias que orienten al estudiante para que se haga consciente del modelo y de lo que este representa en el conocimiento científico.

Las limitaciones se relacionan con aspectos como el tiempo. En esta institución hay opción de que el profesor planee cuidadosamente desde el inicio del año escolar para que se programen contenidos temáticos que sean estructurantes y se puedan desarrollar en los tiempos establecidos para ello. Los procesos administrativos están orientando muchas decisiones que por motivos de poder no son otorgadas a ningún otro estamento.

Smith y Anderson (1984) presentan las dificultades en una experiencia de enseñanza, por ejemplo: ciertas ideas sobre la nutrición de las plantas verdes resultan realmente complicadas para los alumnos teniendo en cuenta las deficiencias acumuladas en los conceptos básicos. Las profesoras recurren al uso de los modelos y simulaciones de manera didáctica, tratan que los estudiantes entiendan algunos organelos celulares, sus funciones y procesos que se dan en las etapas de las fotosíntesis, en ocasiones se utilizan adjetivos o palabras que confunden y no reflejan la realidad. Los modelos pueden generar dificultades de comprensión, el estudiante crea una representación distorsionada de la realidad y mantiene dicha representación; cuando se presenta otro modelo que representa lo mismo, al estudiante le cuesta comprender; también se presenta el caso de aquellos estudiantes quienes le prestan más atención al modelo (color, forma o quien lo hizo) que al proceso que se lleva a cabo en esas estructuras y pierden el hilo del concepto o proceso que se está tratando.

La operatividad del currículo se da en el desarrollo de las clases. Algunos de los elementos de entrada, sobre todo los que se refieren con la planeación, establecimiento de propósitos, logros e indicadores, contenidos temáticos, gradualidad y niveles de complejidad y de evaluación están supeditados al profesor, los controles se dan durante el proceso debido al enfoque que tiene la institución, pero en el caso del grado sexto la cantidad de temáticas fueron establecidas por la profesora al inicio del año escolar, lo que indica que hubo falta de una buena planeación.

En referencia a la pregunta ¿cómo influye el currículo en el desarrollo de la clase?, la profesora Beatriz dijo:

B.:- Influye cuando al planear la clase, es importante cumplir con el objetivo de esa clase, no se puede discutir cuando se sale del tema específico para la clase, el desarrollo de la clase se limita a cumplir con los estándares, con lo básico...esto no promueve la indagación.

La profesora hizo referencia a la poca flexibilidad que tiene el currículo. En cuanto a la ejecución del plan de estudio, el colegio solicita que se abarquen todos los 
contenidos, motivo por el cual el profesor debe asegurar que se desarrollen todas la temáticas, en ocasiones no se tiene en cuenta el advenimiento de situaciones en el aula que requieren de tiempo no estipulado en los documentos.

Ana expresó que el currículo influye en la enseñanza debido que hay muchas temáticas por enseñar en grado sexto, Ana asegura que:

A.:- No se puede profundizar mucho en el tema de la fotosíntesis, ya que durante el periodo se debe enseñar además el sistema respiratorio en animales, nutrición y sistema digestivo. A, C, E-7, R-24.

Esta es una problemática constante, ya que se debe abarcar gran cantidad de temáticas que no permiten el desarrollo profundo de ninguna, por eso es importante que el profesor o el colegio propongan en el plan de estudio unas temáticas estructurantes, abarcadoras y que tengan una pertinencia contextual, a fin de adaptarse a las necesidades cambiantes del entorno, a las posibilidades y a los intereses de los alumnos. Es evidente que en este caso se carece de dicho proceso

Existen otros elementos en la construcción del currículo que no son tenidos en cuenta por ejemplo: los estudiantes, son los profesores y las directivas quienes toman las decisiones, los estudiantes tienen una posición receptiva. La naturaleza del currículo se centra en los contenidos y los procedimientos para comprender la fotosíntesis y las competencias básicas se utilizan como metas de aprendizaje y herramienta para evaluar los mismos. Finalmente las decisiones son de tipo institucional es decir el currículo está pres-escrito por las directivas y es poco flexible. Al igual que los resultados presentados por Charrier (2009) el currículo le da relevancia a la planificación, enuncia objetivos por obligación, centrado en contenidos y procedimientos

El conocimiento experiencial del profesor le permite introducir un repertorio de actividades que probablemente mejoren el desempeño en el aula de clase, siendo las prácticas de laboratorio las preferidas por las profesoras: se evidencia que han ganado experiencia frente a sus habilidades y destrezas en este campo. Es claro en el caso de la profesora que no es licenciada (Beatriz), que hace mayor énfasis en el conocimiento que en la pedagogía para enseñar.

A.:- Me caracterizo por ser una persona responsable, dedicada a los estudiantes, trato de buscar actividades diferentes, me intereso mucho por investigar y por formarme todo el tiempo. A, I, E-8, R-28

La profesora hace énfasis en el nivel social que posee y que la habilita para ejercer la profesión, no incluye en su respuesta características de tipo cognitivo como análisis, síntesis, creatividad, que son necesarias para manejar la multidimensionalidad que se presenta en el aula de clase. 
B.: -Pienso que el conocimiento es el aspecto más relevante hasta ahora, porque, pues, cuando uno sabe lo que está hablando gana como el respeto de las personas que te están escuchando y entonces eso hace que los estudiantes se motiven también por aprender.

El profesor debe contar con un perfil que incluya conocimiento teórico suficientemente profundo y pertinente acerca del aprendizaje, el desarrollo, el comportamiento humano, despliegue de valores, actitudes que fomenten el aprendizaje, relaciones humanas genuinas, dominio de los contenidos o materias que enseña, control de las estrategias de enseñanza que faciliten el aprendizaje del estudiante y lo hagan motivante. Desde su saber, el profesor debe apoyar al estudiante a construir el conocimiento y a ubicarse como actor crítico de su entorno (Loughran, 2001). Además, es importante para un profesional contar con discernimiento, desarrollar hábitos propios de la profesión, tener ética profesional (Tardif, 2004), así como ser dinámico, estratégico, autorregulado y reflexivo.

Es determinante la influencia de la formación inicial con el ejercicio de la profesión, ya que le aporta al campo de la pedagogía y las ciencias de la educación un conocimiento sobre el aprendizaje y la historia que difícilmente se van a adquirir por otro medio.

\section{Conclusiones}

La revisión del marco teórico permitió establecer los elementos del CDCF que se constituyeron en la base de análisis del proceso de enseñanza sobre fotosíntesis de dos profesoras de los grados sexto y noveno. A partir de las propuestas de cuatro autores se establecieron ocho elementos, de los cuales uno emerge de esta investigación: el conocimiento experiencial del profesor. Se consideró enriquecedor indagar acerca de la experiencia, por cuanto las profesoras en la mayoría de decisiones que toman en el aula, en las respuestas y apreciaciones ante las preguntas que se hicieron recurren a lo que ellas han vivido como estudiantes y profesionales, por lo tanto cada uno de los elementos de esta investigación se ven relacionados con el perfil docente, profesional, pedagógico-didáctico con el que cada una de ellas cuenta. Se puede decir que los demás elementos se cimentan en la experiencia que tienen cada una de las profesoras.

En lo que tiene que ver con elementos constitutivos del CDCF de las profesoras Ana y Beatriz, se concluye que:

- En una perspectiva constructivista es básico indagar las ideas y trabajar sobre ellas. En este caso las ideas previas no fueron indagadas al inicio de la clase, pero durante su desarrollo tanto en grado sexto como noveno, se utilizaron diferentes estrategias como analogías, comparaciones, modelos, simulaciones, preguntas, discusiones y trabajos prácticos que despertaron cuestionamientos en los estudiantes y les permitió a las profesoras conocer lo que los estudiantes 
pensaban sobre el tema. Dichas ideas fueron indagadas y manejadas por las profesoras de acuerdo a las temáticas del plan de estudio.

- Con respecto a lo que deben saber los estudiantes antes de la enseñanza de este concepto, ambas profesoras coinciden en que es necesario además de contar con un buen conocimiento biológico, tener bases químicas y físicas. Se hizo más evidente en el grado noveno dicha necesidad por la profundidad del contenido en cuanto a reacciones químicas y ondas electromagnéticas y por la carencia que tenían los estudiantes de vocabulario científico que les permitiera entender los procesos, para dar cuenta de los fotosistemas y el ciclo de Calvin. Los estudiantes se enfrentan a temáticas de las cuales poco se ha construido en términos de relaciones de conceptos, por lo tanto hay debilidad en la adquisición de conocimientos previos para enfrentarse a unos tal vez más complejos.

- Respecto al conocimiento acerca del contenido disciplinar, las dos profesoras tienen razones teleológicas respecto de la importancia de la fotosíntesis, ya que asumen causas finales, la primera para los demás organismos y la segunda para abastecer la economía.

- Las profesoras tienen poco conocimiento de los hechos históricos que han acontecido en cuanto a las investigaciones relacionadas con el proceso. Los profesores tienen la responsabilidad de hacer un recorrido tanto de la aparición del concepto, el estudio del proceso, como de la historia de la enseñanza del concepto, ya que algunas investigaciones han demostrado que las etapas en la historia del desarrollo conceptual se ven reflejadas las formas de aprender de los estudiantes, desde niveles de escolaridad bajos hasta los más altos.

- La manera como las profesoras evalúan el aprendizaje sobre fotosíntesis, difiere a lo planteado en los documentos de planeación y prevalece la memorización y el recuerdo de los conceptos, minoritariamente la aplicación, contextualización e interrelación con situaciones cotidianas. Los proyectos se presentan como una oportunidad para el desarrollo de habilidades donde los estudiantes, por medio de la investigación, deben profundizar lo que están viendo en clase para poder dar cuenta de lo que le pasa a una planta en unas condiciones específicas.

- Es de aclarar, en cuanto a los propósitos de enseñanza, que se relacionan directamente con el conocimiento del contenido disciplinar y el currículo, de este último en particular, con el plan de estudio. El conocimiento sobre la fotosíntesis que tienen las profesoras les permite distinguirlo como un concepto estructurante pero a la hora de la práctica no es tan evidente. Esto muestra los intereses que tienen las profesoras en la enseñanza de la fotosíntesis y la tendencia que tiene el currículo. En ese sentido se debe encontrar las relaciones con otros temas que se listan como obligatorios en los planes de estudio, además determinar el número de clases, la secuencia, gradualidad y articulación por lo menos entre las asignaturas de Física y Química. 
- Una dificultad evidente, se relaciona con el tipo de estrategias que utilizan las profesoras en el aula de clase, ya que se usaron modelos o simulaciones que le exigen al estudiante hacer representaciones de conceptos abstractos con los que no tienen familiaridad. Las imágenes de las cosas que observan en modelos 0 simulaciones impactan más que las palabras o explicaciones que las profesoras utilizan y probablemente la mayoría de estudiantes van a recordar dichas representaciones antes que el contexto o significado científico. Por eso se debe usar un vocabulario acorde con el científico y unos modelos que se acerquen a lo que realmente ocurre en la naturaleza.

- En cuanto al conocimiento experiencial de las profesoras, se puede decir que a las dos les falta conocer el proceso cognitivo que tienen los estudiantes en el proceso de aprendizaje, además que han sido influenciadas en su formación inicial por metodologías que son recurrentes en su labor como profesoras, ambas coinciden en que las prácticas de laboratorio son la mejor manera de aprender, por ende ambas utilizan de diversas maneras los trabajos prácticos para enseñar el concepto de fotosíntesis.

En lo que tiene que ver con la formación docente se puede decir lo siguiente:

En relación con la construcción del CDCF y la necesidad de tenerlo en cuenta en la formación inicial, es de destacar que el análisis del proceso de enseñanza de las dos profesoras es una muestra de la realidad de las aulas de clase y sirve de referencia para aquellos que están en formación, para enfocar sus estudios hacia temáticas que sean estructurantes y que abarquen varios campos de estudio. De esta manera se pueden organizar las acciones desde las diferentes asignaturas (Física, Química y Biología) en el colegio, para que la planeación, ejecución y evaluación le apunten a los mismos objetivos. De esta manera la labor del profesor debe estar encaminada a trabajar en equipo para propender por la comprensión de conceptos y la mejora de la enseñanza.

\section{BIBLIOGRAFÍA}

ÁLVAREZ, J.M. (2007). Evaluación: entre la simplificación técnica y la práctica crítica. Novedades Educativas. 18 (195), 8-10.

ARNAL, J. y OTROS. (1992). Investigación educativa. Fundamentos y metodología. Barcelona, España: Editorial Labor.

ASIMOV, I. (1980). Fotosíntesis. España: Plaza y Janes Editores.

ASTOLFI, J. (2001). Transposición didáctica. En: Conceptos claves en la didáctica de las disciplinas. Referencias, definiciones y bibliografías de didáctica de las ciencias. Traducción de Pabla Manzano. Sevilla: Díada.

AUDESIRK, T., AUDESIRK, G. (2001). Biology. Life on Earth. Prentice Hall. U.S.A. 
BAKER, J, Y ALLEN. G. (1970) Biología e Investigación científica. Fondo Educativo Interamericano. EUA. Washington D. C. p. 666

BROMME, R. (1988). Conocimientos profesionales de los profesores. Enseñanza de las Ciencias, 6 (1), 19-29.

CAÑAL, P. (2004). La enseñanza de la biología: ¿Cuál es la situación actual y qué hacer para mejorarla? Alambique. Didáctica de las ciencias experimentales, $N^{\circ} 41$, pp. 27-41.

CARLSEN, W. (1987). Why do you ask? The Effects of Science Teacher Subject Matter Knowledge on Teacher Questioning and Classroom Discourse. Paper presented at the annual meeting of the American Educational Research Association.

CARLSEN, W. (1999). Domains of Teacher Knowledge. In: GESS-NEWSOME, J. and LEDERMAN, N. (Eds.). Examining Pedagogical Content Knowledge. The Construct and its Implications for Science Education. Dordrecht, Boston, London: Kluwer Academic Publishers. pp. 133-144.

CHARRIER, M. (2005). El conocimiento didáctico del contenido sobre la fotosíntesis. Estudio de las concepciones y de la práctica de los profesores del tercer ciclo de la educación general básica argentina. Tercer Encuentro de Investigadores en Didáctica de la Biología Extraído el 29 de noviembre de 2009, de http://www.adbia.com.ar/eidibi archivos/aportaciones/paneles/trabajos completos/ch arrier tesis.pdf [Consulta Noviembre 29 de 2009].

CHARRIER, M. (2009). El conocimiento didáctico del contenido sobre la fotosíntesis de dos profesores de educación secundaria argentinos. Enseñanza de las Ciencias. Número Extra VII congreso internacional sobre investigación en la didáctica de las ciencias. Barcelona, pp. 2585-2590 Extraído el 20 de enero de 2011, de http://ensciencias.uab.es/congrso09/numeroextra/art-2585-2590.pdf.

CHÁVEZ, M. (2010). Estudio analítico no lineal de los modelos explicativos de la nutrición vegetal y su valor para el proceso de enseñanza aprendizaje. Digitalizado en Red Académica. Extraído el 15 de enero, 2011, de http://www.pedagogica.edu.co/storage/ted/articulos

DÍAZ, F., BARRIGA, A., HERNÁNDEZ, G. (2003). Estrategias docentes para un aprendizaje significativo. México: 2da Edición. McGraw-Hill.

EISEN, Y., STAVY, R, (1992). Material Cycles in Nature. A New Approach to Photosynthesis in Junior High School. The American Biology Teacher. 54 (6), 339342. 
FINLEY, A., STEWART, J. y YARROCH W. L. (1982). Teachers Perceptions of Important and Difficult Science Content. Science Education, 66 (4), 531-538.

GENÉ, A. y ASTUDILLO, H., (1984). Errores conceptuales en Biología: la fotosíntesis de las plantas verdes, Enseñanza de las Ciencias, 2, 15-16.

GESS-NEWSOME, J. (1999). Pedagogical Content Knowledge: An introduction and orientation. En: GESS-NEWSOME, J. and LEDERMAN, N. (Eds.). Examining Pedagogical Content Knowledge. The construct and its implications for Science education. Dordrecht, Boston, London: Kluwer Academic Publishers. 3-17.

GÓMEZ, J. (2000). Competencias problemas conceptuales y cognitivos. Socolpe. Bogotá. p.126.

GONZÁlEZ, C., GARCÍA, S., MARTínEZ, C. (2003). ¿A qué contenidos relacionados con la fotosíntesis dan más importancia los textos escolares de secundaria? Enseñanza de las ciencias. Número extra. pp. 77-88.

GROSSMAN, P. (1990). The making of a teacher knowledge and teacher education, New York: Teachers college, Columbia University.

JOHNSTONE, A.H. y MAHMOUD, N.A. (1980). Isolating topics of high perceived difficulty in school biology. Journal of Biological Education, 14 (2), 163-166.

LORENZO, M. y FARRÉ, A. 2009. El análisis del discurso como metodología para reconstruir el Conocimiento Didáctico del Contenido. Enseñanza de las Ciencias. VIII congreso internacional sobre investigación en la didáctica de las ciencias (ISSN 0212-4521) Extraído el 8 de agosto de 2010, de: http://ensciencias.uab.es

LOUGHRAN, J. (2001). Documenting Science Teachers' Pedagogical Content Knowledge through Papers. Research in Science Education 31: 289-307.

MAGNUSSON, S.; KRAJCIK, J. and BORKO, H. (1999). Nature, Sources, and Development of Pedagogical Content Knowledge for Science Teaching. In: GESSNEWSOME, J. and LEDERMAN, N. (Eds.). Examining Pedagogical Content Knowledge. The Construct and its Implications for Science Education. Dordrecht, Boston, London: Kluwer Academic Publishers. 95-132.

MARCELO, C. (1999). Cómo conocen los profesores la materia que enseñan. Algunas contribuciones de la investigación sobre conocimiento didáctico del contenido. En: MONTERO, L. y J. M. VET, J.M. (Eds.). Las didácticas especificas en la formación del profesorado. Santiago, Tórculo; 151-185.

MAYR, E. (2006). ¿Por qué es única la Biología? Consideraciones sobre la autonomía de una disciplina científica. Argentina: Ed. Katz. 
MEN. (2006). Estándares Básicos de Competencias en Lenguaje, Matemáticas, Ciencias y Ciudadanas. Editor Ministerio de Educación Nacional. Bogotá Colombia. p.95.

MEN. (2008). Verificación de los requisitos básicos de funcionamiento de programas de formación para el trabajo y el desarrollo humano. Editor Ministerio de Educación Nacional. p. 6.

OSBORNE R., FREYBERG, P. (1995). Influencia de las "ideas previas" de los alumnos. Segunda Edición. España: Ediciones. Narcea.

PORLÁN, R., RIVERO GARCÍA, A., y MARTÍN DEL POZO, R. (1997). Conocimiento profesional y epistemología de los profesores I: teoría, métodos e instrumentos. Enseñanza de las Ciencias 15(2), 155-171.

PORLÁN, R.; RIVERO, A. y MARTíN DEL POZO, R. (2000). El conocimiento del profesorado sobre la ciencia, su enseñanza y aprendizaje. En: PERALES, F. y CAÑAL. P. (Compiladores). Didáctica de las Ciencias Experimentales. Teoría y práctica de la Enseñanza de las Ciencias. Alcoy: Marfil. 507-533.

POZO, J.I. (1987). Aprendizaje de la ciencia y conocimiento causal. Madrid: Aprendizaje/Visor. 295.

QUINTANILLA, M., DAZA, S., MERINO, C. (2010). Unidades Didácticas en Biología y educación ambiental. Su contribución a la promoción de competencias de pensamiento científico. Volumen 4. Fondecyt. Extraído el 24 de abril, 2011, de: http://www.uc.cl/sw educ/educacion/grecia/plano/html/pdfs/destacados/LibroDBioGre cia11julio.pdf

SHULMAN, L. (1986). Those who understand: Knowledge growth in teaching. Educational Researcher, 15 (2), 4-14.

SHULMAN, L.S. (1987). Knowledge and teaching: Foundations of the new reform. Harvard Educational Review, 57, 1-22.

SMITH, E, y ANDERSON, C. (1984). Plants as producers: A case of elementary science teaching. Journal of research in science teaching, 21(7), 105-115.

STONE, M. (1998). La enseñanza para la comprensión. Vinculación entre la investigación y la práctica. Buenos Aires. Argentina: Ed. Paidos.

TARDIF, M. (2004). Los saberes del docente y su desarrollo profesional. Traducción de Pablo Manzano. Madrid: Narcea.

TEST, D. W y WEWARD, W, L. (1980). Photosynthesis: teaching a complex science concept to juvenile delinquents. Science Education, 64, 129-139. 
VALBUENA, E. (2007). El conocimiento didáctico del contenido biológico. Estudio de las concepciones disciplinares y didácticas de futuros docentes de la Universidad Pedagógica Nacional (Colombia). Tesis doctoral. Universidad Complutense de Madrid

WANDERSEE, J.W. (1983). Students misconceptions about photosynthesis: a crossage study. In H. Helm \& J.D. Novak (Eds.). Proceedings of the International Seminar: Misconceptions in Science and Mathematics, 441-446 Ithaca, NY: Cornell University. 